\title{
Propuesta y validación de escalas para la integración externa con proveedores y clientes. \\ Una aplicación en la industria textil valenciana
}

\section{Proposal and validation of scales for external integration with suppliers and customers. An application in the valencian textile industry}

\author{
Manuel Expósito-Langa ${ }^{3}$, Débora Nicolau-Juliá ${ }^{3}$ y Teresa Vallet-Bellmunt ${ }^{4}$ \\ ${ }^{3}$ Departament d' Organización d'Empreses. Universidad Politécnica de Valencia. \\ Escuela Politécnica de Alcoy, Pl. Ferrándiz i Carbonell, 2 Alcoi- Alicante. \\ ${ }^{4}$ Departamento de Administración de Empresas y Marketing. Universidad Jaume I. \\ Grupo de Investigación AERT. Unidad Asociada INGENIO (CSIC-UPV)
}

maexlan@doe.upv.es

Fecha de recepción: 22-6-2010

Fecha de aceptación: 10-11-2010

Resumen: La integración externa refleja el grado en que las actividades logísticas de una empresa están interrelacionadas con las de sus proveedores y clientes dentro de la cadena de suministro. Sin embargo, se observa que no existe un consenso a la hora de medir dicho concepto. De esta forma, a partir de una revisión de la literatura, pretendemos realizar una aportación a este debate mediante una propuesta y depuración de una escala para la integración externa, tanto con proveedores como con clientes. El trabajo ha sido validado en la industria textil valenciana.

Palabras clave: integración externa, cadena de suministro, industria textil, análisis factorial confirmatorio.

Abstract: The external integration reflects the extent to which the logistics activities of a firm are interrelated with their suppliers and customers within the supply chain. However, it appears that there is no consensus in measuring this concept. Thus, from a literature review, we intend to make a contribution to this debate with a proposal and treatment of a scale for external integration with both suppliers and clients. The work has been validated in the Textile Industry in Valencia.

Key words: external integration, supply chain, textile industry, confirmatory factor analysis.

\section{Introducción}

Durante la última década, la literatura sobre cadena de suministro ha puesto un especial énfasis en la integración empresarial, tanto interna como externa, como un factor clave para el logro de mejoras competitivas (Mittermayer y Rodríguez, 2009; Vallet-Bellmunt, 20l0). En el caso particular de la integración externa, ésta refleja el grado en que las actividades logísticas de una empresa están interrelacionadas con las actividades logísticas de sus proveedores, clientes y otros miembros de la cadena de suministro (Frohlich y Westbrook, 200I). De esta forma, numerosos autores (Frohlich y Westbrook, 200 I; Droge et al. 2004; Giménez y Ventura, 2005) parecen estar de acuerdo con que un elevado nivel de integración tendría un impacto positivo sobre los resultados de las empresas implicadas. Sin embargo, otros autores tienen dudas al respecto y sugieren que esta relación es más retórica que real (Van derVaart y Van Donk, 2008; Fabbe-Costes y Jahre, 2007), ya que, entre otras cuestiones, existe poco consenso sobre cómo capturar la esencia de la integración, así como los tipos de resultados sobre los que se mide su influencia.

Esta falta de consenso en la literatura sobre la manera de medir el concepto de la integración externa nos lleva a proponer y validar en el presente trabajo una escala de medida para la integración externa, dentro del contexto de la cadena de suministro.

El trabajo está estructurado de la siguiente manera: en primer lugar hemos realizado una revisión bibliográfica del concepto de la integración externa, con el 
fin de determinar aquellas aportaciones más relevantes que nos permitan sintetizar y establecer una escala de medida; a continuación, describiremos el sector textil valenciano como objeto de estudio; seguidamente validaremos la escala mediante la aplicación de un análisis factorial confirmatorio, y para finalizar presentaremos las conclusiones del trabajo.

\section{Marco Teórico}

\section{I. El concepto de la Integración Externa}

La integración logística en la empresa viene caracterizada tanto por la integración de las actividades internas o entre departamentos funcionales dentro de la misma, como por la integración externa de las actividades logísticas con las de otros miembros de la cadena de suministro, más allá de las fronteras entre empresas (Stock et al., 1998).

En este trabajo nos hemos centrado en el concepto de integración externa, que aparece en la bibliografía como una evolución natural del concepto de integración interna, existiendo a su vez cierto consenso en que la integración externa se produce como una segunda fase, cuando la empresa tiene ya integradas sus funciones logísticas internas (Stevens, 1989; Giménez y Ventura, 2003, 2005).

La integración externa de la empresa tiene lugar a lo largo de todo el canal de comercialización, es decir, a través de las etapas por las que pasa un producto desde su origen (producción) hasta su destino (consumo) (Sánchez, 1997; Cuello et al., 2003). De esta forma, entendemos la idea de canal como cadena de suministro, y la integración externa como el conjunto de relaciones verticales interorganizacionales. Es decir, la integración externa hace referencia al conjunto de las relaciones verticales entre todas las empresas que participan en la producción, distribución, manipulación, almacenamiento y comercialización de un producto y sus componentes. La gestión de dicho canal supondrá que las distintas empresas dejen de intentar mejorar sus propios procesos de forma independiente para alcanzar un beneficio global mediante colaboración (Vallet-Bellmunt, 20l0).

Por otro lado, a la hora de abordar el estudio de la integración externa es importante considerar el número de empresas que integran la cadena de suministro. Identificamos diferentes posicionamientos, así por ejemplo, Christopher (1998) incluye la gestión de las relaciones ascendentes y descendentes con pro- veedores y clientes llevadas a cabo para conseguir un valor superior para el consumidor y un menor coste en global. Por otro lado, Mentzer et al. (200I) proponen un conjunto de tres o más entidades (organizaciones o individuos) directamente relacionados con los flujos ascendentes o descendentes de bienes, servicios, finanzas o información, desde el origen hasta el consumidor final. Por último, Bagchi y Skjoett-Larsen (2005) distinguen tres tipos de cadenas de suministro: I) La cadena de suministro directa que incluye la organización con un proveedor y un cliente; 2 ) La cadena de suministro extendida que incluye el proveedor del proveedor y el cliente del cliente; y 3) La cadena última que incluye todas las organizaciones relacionadas con la cadena, como por ejemplo, los operadores logísticos, proveedores financieros o proveedores de servicios de gestión.

Adicionalmente, se observan diferentes formas de trabajar la integración externa, así algunos autores entienden la integración como una red de empresas interactuando entre sí para entregar un producto de mayor valor al consumidor (Lejeune y Yakona, 2005). Por otro lado, Narasimhan y Das (200I) analizan el desarrollo de la relación del comprador-proveedor a través de diferentes indicadores como por ejemplo la confianza. Frohlich y Westbrook (200 I) y Rosenzweig et al. (2003) determinan tanto la intensidad de la integración hacia proveedores y clientes. Rodrigues et al. (2004) parten del la idea de la colaboración. Stank et al. (2004), Vickery et al. (2004), y Li et al. (2006) utilizan tiempos de respuesta. Por último, Van der Vaart y Van Donk (2008) realizan un análisis detallado de los ítems utilizados en la literatura para medir la integración y llegan a distinguir tres categorías de escalas: las que miden actitudes, las que miden prácticas y las que miden patrones de comportamiento.

Por tanto, para hacer operativo el concepto de la integración encontramos en la literatura múltiples indicadores relacionados con aspectos como la intensidad de la comunicación, la coordinación de las actividades, la creación de equipos, la ausencia de fronteras rígidas entre las actividades logísticas de las empresas y las de los proveedores o clientes, la elaboración de decisiones conjuntas, o el intercambio de información. Esta excesiva variación en la forma en que los autores miden el concepto de la integración queda plasmada en la Tabla I, donde reflejamos aquellos trabajos más relevantes en los que se ha estudiado y medido el concepto de la integración externa, y que serán fundamentales para nuestro objetivo. 
Tabla I

Trabajos relativos a la Integración Externa

\begin{tabular}{|c|c|c|c|}
\hline Autor/es & Muestra & Escala de la Integración Externa & ĺtems \\
\hline $\begin{array}{l}\text { Dean, J.W.y Snell, } \\
\text { S. (|99|) }\end{array}$ & $\begin{array}{l}\text { Gerentes y empleados } \\
\text { de empresas de la in- } \\
\text { dustria del metal en Pen- } \\
\text { silvania. }\end{array}$ & Creación propia. Validada con 5 ítems. & $\begin{array}{l}\text { I. Número de proveedores, } 2 \text {. Tamaño de } \\
\text { sus entregas, 3. Duración del producto que } \\
\text { se trabaja, } 4 \text {. Número total de piezas que } \\
\text { se proveen, } 5 \text {. Importe del Stock de con- } \\
\text { trol existente. }\end{array}$ \\
\hline $\begin{array}{l}\text { Miller, J.G. y Roth, } \\
\text { A.V. (1994) }\end{array}$ & $\begin{array}{l}\text { Encuestas las unidades } \\
\text { de negocio de diferentes } \\
\text { centros de fabricación. }\end{array}$ & $\begin{array}{l}\text { A partir del trabajo realizado por la Uni- } \\
\text { versidad de Boston. Validada con I I } \\
\text { ítems. }\end{array}$ & $\begin{array}{l}\text { I. Bajo precio, 2. Flexibilidad en el diseño, } \\
\text { 3. Flexibilidad en volumen, 4. Conformidad, } \\
\text { 5. Rendimiento, 6. Velocidad, 7. Confianza } \\
\text { en la entrega, 8. Servicio post-venta, 9. Pu- } \\
\text { blicidad, I0. Amplia distribución, II. Am- } \\
\text { plia línea de productos. }\end{array}$ \\
\hline $\begin{array}{l}\text { Ellinger, A.E., } \\
\text { Daugherty, P.J. y } \\
\text { Keller, S.B. (2000) }\end{array}$ & $\begin{array}{l}\text { Directores logísticos de } \\
\text { empresas de fabricación } \\
\text { Americanas. }\end{array}$ & $\begin{array}{l}\text { A partir de investigaciones anteriores } \\
\text { y sugerencias recibidas durante la reali- } \\
\text { zación de las pruebas. Validada con } 7 \\
\text { ítems. }\end{array}$ & $\begin{array}{l}\text { I. Cooperación para lograr objetivos, } \\
\text { 2. Desarrollo de un entendimiento mutuo } \\
\text { sobre responsabilidades, 3. Equipo de tra- } \\
\text { bajo informal, 4. Compartir ideas, infor- } \\
\text { mación y recursos, 5. Trabajar conjunta- } \\
\text { mente como un equipo, 6. Planificación } \\
\text { conjunto para anticipar y resolver proble- } \\
\text { mas operativos, 7.Toma de decisiones con- } \\
\text { juntas para una mejor rentabilidad. }\end{array}$ \\
\hline $\begin{array}{l}\text { Shin, H., Collier, } \\
\text { D.A.yWilson, D.D. } \\
(2000)\end{array}$ & $\begin{array}{l}\text { Empresas estadouniden- } \\
\text { ses dedicadas a la fabri- } \\
\text { cación de automóviles. }\end{array}$ & Creación propia. Validada con 5 ítems. & $\begin{array}{l}\text { I. Coste del proveedor, } 2 \text {. Confianza en la } \\
\text { entrega del proveedor, 3.Tiempo que tar- } \\
\text { da el proveedor en realizar la entrega, } 4 \text {. El } \\
\text { proveedor realiza la entrega a tiempo, } \\
\text { 5. Calidad del proveedor. }\end{array}$ \\
\hline $\begin{array}{l}\text { Narasimhan, R. y } \\
\text { Das, A. (200I). }\end{array}$ & $\begin{array}{l}\text { Gerentes de compras de } \\
\text { las empresas de fabrica- } \\
\text { ción de metal, equipa- } \\
\text { mientos informáticos, } \\
\text { electrónicos, transporte } \\
\text { de equipos y artículos de } \\
\text { óptica. }\end{array}$ & $\begin{array}{l}\text { A partir de los trabajos de Miller y Roth } \\
\text { ( } 1994) \text {, Dean y Snell ( } 1991 \text { ), y creación } \\
\text { propia. Validada con } 6 \text { ítems. }\end{array}$ & $\begin{array}{l}\text { I. Relación de subcontratación de la em- } \\
\text { presa con el principal proveedor (asocia- } \\
\text { ción de equipo), 2. El grado de confianza } \\
\text { mutua, 3. El compromiso de la alta direc- } \\
\text { ción en el desarrollo de la relación, } 4 \text {. Re- } \\
\text { solución de problemas conjuntamente con } \\
\text { el proveedor, } 5 \text {. Se informa al proveedor } \\
\text { del calendario de producción, } 6 \text {. El prove- } \\
\text { edor informa a la empresa de su calenda- } \\
\text { rio de producción. }\end{array}$ \\
\hline $\begin{array}{l}\text { Stank, T.P., Keller, } \\
\text { S.B. y Closs, D.J. } \\
\text { (200I-2002). }\end{array}$ & $\begin{array}{l}\text { Empresas fabricantes, ma- } \\
\text { yoristas, minoristas y dis- } \\
\text { tribuidores de la industria } \\
\text { automotriz, química in- } \\
\text { dustria de consumo du- } \\
\text { radero, elaboración de } \\
\text { alimentos, venta al por } \\
\text { menor en general y papel. }\end{array}$ & $\begin{array}{l}\text { Medidas desarrolladas por el equipo de } \\
\text { investigadores de la Universidad estatal } \\
\text { de Michigan. Validada con } 6 \text { ítems. }\end{array}$ & $\begin{array}{l}\text { I. Mi empresa define claramente sus fun- } \\
\text { ciones y responsabilidades con los socios } \\
\text { de la cadena de suministro, } 2 \text {. Mi empresa } \\
\text { está Ilevando a cabo un plan para esta- } \\
\text { blecer alianzas y asociaciones, 3. Mi em- } \\
\text { presa está dispuesta a compartir informa- } \\
\text { ción estratégica con proveedores y } \\
\text { clientes clave, } 4 \text {. La empresa está dispues- } \\
\text { ta a compartir los resultados con los pro- } \\
\text { veedores y clientes clave, } 5 \text {. Mi empresa si- } \\
\text { gue unas pautas para el desarrollo, } \\
\text { mantenimiento y seguimiento de las rela- } \\
\text { ciones en la cadena de suministro, } 6 \text {. Mi } \\
\text { empresa tiene acuerdos con proveedores } \\
\text { y clientes que operan bajo los principios } \\
\text { de recompensa y riesgos compartidos. }\end{array}$ \\
\hline
\end{tabular}


Tabla I

Trabajos relativos a la Integración Externa (continuación)

\begin{tabular}{|l|l|l|l|}
\hline \multicolumn{1}{|c|}{ Autor/es } & \multicolumn{1}{|c|}{ Muestra } & Escala de la Integración Externa & \multicolumn{1}{c|}{ Ítems } \\
\hline $\begin{array}{l}\text { Rosenzweig, E.D., } \\
\text { Roth, A.V.y Dean, }\end{array}$ & $\begin{array}{l}\text { Empresas situadas en el } \\
\text { primer cuartil por sus } \\
\text { ventas según un proyec- } \\
\text { to de la industria manu- } \\
\text { facturera realizado por la } \\
\text { universidad de Carolina } \\
\text { del norte. }\end{array}$ & $\begin{array}{l}\text { A partir del trabajo de Stevens (1989) } \\
\text { y creación propia.Validada con 4 ítems. }\end{array}$ & $\begin{array}{l}\text { I. Estrechamente integrado con la organi- } \\
\text { zación, 2. Estrechamente integrado con los } \\
\text { proveedores de materias primas, 3. Estre- } \\
\text { chamente ligado con distribuidores y mi- } \\
\text { noristas, 4. Estrechamente ligado con los } \\
\text { clientes. }\end{array}$ \\
\hline
\end{tabular}

\section{Trabajo Empírico}

\section{I. La industria textil valenciana}

En este trabajo estudiaremos la industria textil, un sector heterogéneo y diverso que comprende un amplio número de actividades productivas, desde la producción de las fibras, hasta la elaboración de la prenda de vestir o artículo final que llega al consumidor. Se trata de un sector considerado de demanda débil y contenido tecnológico bajo, y viene caracterizado por un proceso productivo intensivo en mano de obra, especialmente en la actividad de confección.

El sector textil español se compone principalmente de PYME de origen familiar, distribuidas por todo el territorio, donde las mayores concentraciones se producen en el arco mediterráneo, concretamente en la ComunidadValenciana y Cataluña, mientras que otras comunidades como Castilla la Mancha, Andalucía o Galicia poseen también un desarrollo, aunque menor, significativo. Este sector tiene un peso relativo importante en la industria española, ya que según datos del Consejo Intertextil Español (CITYC) durante 2008 ha supuesto aproximadamente un 7\% del total del empleo industrial, así como una contribución al PIB español cercana al 4\%.

Centrándonos de forma particular en la industria textil valenciana como objeto de estudio, ésta se encuentra situada principalmente en las comarcas de L'Alcoià, El Comtat y L'Alt Vinalopó en Alicante y La Vall d'Albaida en Valencia. Según la Asociación de Empresarios Textiles de la Comunidad Valenciana (ATEVAL), estas comarcas han generado durante 2008 el 17\% de la producción textil en España ( I.825 millo- nes de $€)$, con una ocupación directa de algo más de 32.000 trabajadores. Los principales productos que se fabrican son los textiles para el hogar, junto a un segmento de productos, denominados textiles de uso técnico, que está creciendo progresivamente en estos últimos años.

\subsection{Confección de la muestra}

Tal y como hemos comentado anteriormente, nuestro estudio empírico se ha centrado sobre una muestra de empresas pertenecientes al sector textil de la ComunidadValenciana. Para la confección de la muestra se ha considerado el total de la población objeto de estudio. La identificación de la misma se ha realizado a partir de los registros del año 2008 de la base de datos SABI I

El listado inicial de empresas se depuró eliminando aquéllas que bien por su reducido tamaño, o por el tipo de producto, no se consideraron significativas del sector, ya que podían mostrar una excesiva heterogeneidad. A su vez, la información de SABI sirvió para establecer un control de algunas respuestas recogidas.

El trabajo de campo se extendió durante los meses de junio a septiembre de 2009 y la fuente de información básica utilizada fue un cuestionario con escala Likert I a 7. Con anterioridad a su distribución, se realizó un cuestionario piloto que fue completado por 5 empresas seleccionadas del sector en función de su trayectoria e importancia en el mismo para la redefinición y ajuste de las variables e indicadores. El cuestionario final fue formulado tras solicitar también la opinión de un panel de expertos.

\footnotetext{
I SABI es un directorio de empresas españolas y portuguesas que recoge información general y datos financieros.
} 
La realización del trabajo de campo se desarrolló en base a entrevistas con los directivos de las empresas seleccionadas a partir de la encuesta diseñada. Se obtuvieron 156 entrevistas válidas, representando un margen de error muestral del $\pm 7,1 \%$ con un intervalo de confianza del 95,5\%.

A continuación, en la Tabla 2 mostramos la ficha técnica del trabajo:

Tabla 2

Ficha técnica de la investigación

\begin{tabular}{|c|c|}
\hline UNIVERSO & $\begin{array}{c}\text { Empresas pertenecientes al sector } \\
\text { textil }\end{array}$ \\
\hline ÁMBITO GEOGRÁFICO & Comunidad Valenciana \\
\hline MARCO MUESTRAL & 739 empresas del sector textil \\
\hline ELEMENTO MUESTRAL & $\begin{array}{c}\text { Director de logística/ Director } \\
\text { de operaciones }\end{array}$ \\
\hline TAMAÑO MUESTRAL & $\begin{array}{l}56 \text { empresas del sector textil, con } \\
\text { un índice de respuesta del } 21,1 \%\end{array}$ \\
\hline ERROR MUESTRAL & $\begin{array}{c} \pm 7,1 \% \\
\text { Nivel de confianza del } 95 \% \\
z=1,96 ; p=q=0,5\end{array}$ \\
\hline DISEÑO MUESTRAL & $\begin{array}{c}\text { Diseño y Pre-tests cuestionario. } \\
\text { Encuesta. }\end{array}$ \\
\hline TRABAJO DE CAMPO & Junio a septiembre de 2009 \\
\hline $\begin{array}{l}\text { INFORMACIÓN } \\
\text { RECOGIDA }\end{array}$ & $\begin{array}{l}\text { Datos básicos de la empresa, Inte- } \\
\text { gración externa con proveedores, } \\
\text { Integración externa con clientes. }\end{array}$ \\
\hline
\end{tabular}

\subsection{Medida de la integración externa con proveedores y clientes}

Para hacer operativo el concepto de la integración externa y poder adaptarlo al objeto de esta investigación, se propone una escala de siete ítems vinculados a distintas actividades que comúnmente utilizan las empresas para integrarse con proveedores y clientes. Por esto, plantearemos dos escalas similares particularizadas para proveedor y cliente.

Los ítems proceden de la revisión de investigaciones anteriores. De esta forma, a partir de la idea de la existencia de equipos informales planteada en los trabajos de Ellinger et al. (2000), Narasimhan y Das (200I), Rosenzweig et al. (2003), Rodrigues et al. (2004), Giménez y Ventura (2005) y Li et al. (2006), se propone el ítem I (Existen equipos informales con los proveedores/clientes clave para integrar procesos). Por lo que respecta al ítem 2 (Se comparten ide- as, información y otros recursos con los proveedores/clientes clave), está relacionado con la idea de compartir información sobre ventas y niveles de existencias y lo encontramos en trabajos como los de Ellinger et al. (2000), Narasimhan y Das (200 I), Stank et al. (200 I), Giménez y Ventura (2005) y Li et al. (2006). El ítem 3 (Existen equipos formales con los proveedores/clientes clave para integrar procesos) está relacionado con la idea del desarrollo conjunto de procesos logísticos y lo podemos encontrar en trabajos como los de Ellinger et al. (2000), Narasimhan y Das (200 I), Stank et al. (200I), Giménez y Ventura (2005), Rodrigues et al. (2004) y Li et al. (2006). A partir de la idea de una planificación conjunta para resolver problemas operativos planteada en los trabajos de Ellinger et al. (2000), Narasimhan y Das (200 I), Stank et al. (200I) y Giménez y Ventura (2005), se propone el ítem 4 (Se realiza una planificación conjunta con los proveedores/clientes clave para anticipar y resolver problemas). Por otro lado, de la idea de incluir a los proveedores y clientes en el establecimiento de objetivos y planificación de las actividades, planteada en los trabajos de Ellinger et al. (2000), Stank et al. (200 I), Giménez y Ventura (2005) y Li et al. (2006) desarrollamos el ítem 5 (Se establecen objetivos conjuntos con los proveedores/clientes clave). Por su parte, el ítem 6 (Se desarrollan responsabilidades conjuntas con los proveedores/clientes clave) está relacionado con la idea de que la empresa sigue unas pautas para el desarrollo, mantenimiento y seguimiento en las relaciones a lo largo de la cadena de suministro, y lo podemos encontrar en los trabajos de Ellinger et al. (2000), Stank et al. (200I), Giménez y Ventura (2005) y Li et al. (2006). Por último, el ítem 7 (Se toman decisiones conjuntas con los proveedores/clientes clave para mejorar la eficiencia de los costes) se desarrolla a partir de la idea de tomar decisiones conjuntas acerca de las mejores maneras para conseguir la eficiencia en costes y lo podemos encontrar en los trabajos de Stank et al. (200I), Giménez y Ventura (2005) y Li et al. (2006).

En la Tabla 3 se muestran los ítems (medidos en una escala Likert de I a 7, donde I = nunca o totalmente en desacuerdo y $7=$ siempre o totalmente de acuerdo).

\section{Resultados}

\section{I. El proceso de validación de escalas}

La validez de una escala hace referencia al grado en el cual el proceso de medida está libre tanto de errores sistemáticos como de errores aleatorios (Kinnear 
Tabla 3

Ítems utilizados en la medida de la integración externa con proveedores y clientes

\begin{tabular}{|ll|}
\hline \multicolumn{1}{|c|}{ Integración externa con proveedores } \\
\hline I. & Existen equipos informales con los proveedores clave para integrar procesos \\
\hline 2. & Se comparten ideas, información y otros recursos con los proveedores clave \\
\hline 3. & Existen equipos formales con los proveedores clave para integrar procesos \\
\hline 4. & Se realiza una planificación conjunta con los proveedores clave para anticipar y resolver problemas \\
\hline 5. & Se establecen objetivos conjuntos con los proveedores clave \\
\hline 6. & Se desarrollan responsabilidades conjuntas con los proveedores clave \\
\hline 7. & Se toman decisiones conjuntas con los proveedores clave para mejorar la eficiencia de los costes \\
\hline 1. & Existen equipos informales con los clientes clave para integrar procesos \\
\hline 2. & Se comparten ideas, información y otros recursos con los clientes clave \\
\hline 3. & Existen equipos formales con los clientes clave para integrar procesos \\
\hline 4. & Se realiza una planificación conjunta con los clientes clave para anticipar y resolver problemas \\
\hline 5. & Se establecen objetivos conjuntos con los clientes clave \\
\hline 6. & Se desarrollan responsabilidades conjuntas con los clientes clave \\
\hline 7. & Se toman decisiones conjuntas con los clientes clave para mejorar la eficiencia de los costes \\
\hline
\end{tabular}

y Taylor, 1995). Desde el trabajo seminal de Anderson y Gerbing (1988) la creación de una escala exige un proceso de contrastación de las propiedades psicométricas del instrumento de medida. Así, no es suficiente sólo con comprobar la fiabilidad mediante el Alpha de Cronbach, sino que es exigible, al menos, la realización de un análisis factorial confirmatorio, que nos permitirá validar la escala y confirmar que supone una buena medida de los constructos que se quieren evaluar. De esta forma, hemos desarrollado un análisis factorial confirmatorio que especifique las relaciones de las medidas observadas. Si este modelo proporciona un mal ajuste, será necesaria la re-especificación hasta obtener unos indicadores de bondad de ajuste que nos garanticen la unidimensionalidad del constructo. Las pruebas se han realizado con el software EQS v. 6.I.

\subsection{Análisis confirmatorio de la escala de integración externa}

Previamente, y como señala Bollen (1989), hay que asegurar la validez de contenido de las escalas, si bien ésta constituye una evaluación subjetiva que depende del investigador, no existiendo, por tanto, un criterio objetivo. En nuestro caso las escalas han sido desarrolladas siguiendo la metodología habitual del examen de investigaciones previas cuyas propiedades han sido testadas a lo largo de la literatura, lo que confirmaría dicha validez de contenido.

A continuación, con el objeto de depurar y validar las escalas utilizadas para la integración externa con proveedores y clientes se ha aplicado el desarrollo de modelos estructurales (Hair et al., 1999), realizando las pruebas pertinentes para medir su validez de contenido, su unidimensionalidad, y su consistencia interna (Frohlich y Westbrook, 200I). Este análisis de los datos se lleva a cabo con un estudio de la dimensionalidad y consistencia interna de las escalas mediante el análisis factorial confirmatorio (AFC). Mediante esta técnica iremos eliminando de forma progresiva y de uno en uno aquellos ítems que incumplan los criterios establecidos en Jöreskog y Sörbom (1993), resultando cada vez en un nuevo modelo factorial.

El primer AFC, mostrado en la Tabla 4, nos muestra que hay algunos ítems con una carga factorial inferior a $(0,7)$, lo que implicaría que esos indicadores son no sustanciales ya que no cumplen el criterio de convergencia fuerte (Jöreskong, y Sörbom, 1993) en ambas escalas. Por otro lado, valores de ajuste como 
Tabla 4

Análisis factorial de las siete actividades de integración e indicadores de fiabilidad

\begin{tabular}{|c|c|c|c|c|c|}
\hline & \multirow[b]{2}{*}{ Análisis factorial con 7 ítems } & \multicolumn{2}{|c|}{$\begin{array}{l}\text { Integración externa } \\
\text { con proveedores }\end{array}$} & \multicolumn{2}{|c|}{ Integración externa con clientes } \\
\hline & & $\begin{array}{c}\text { Carga } \\
\text { factorial }(\lambda)\end{array}$ & Fiabilidad & $\begin{array}{c}\text { Carga } \\
\text { factorial }(\lambda)\end{array}$ & Fiabilidad \\
\hline I. & $\begin{array}{l}\text { Existen equipos informales con los pro- } \\
\text { veedores/clientes clave para integrar } \\
\text { procesos }\end{array}$ &, 413 & \multirow{7}{*}{$\begin{array}{c}X^{2}=69,756 \\
\text { P-valor }=, 000 \\
\text { GFI }=, 875 \\
\text { AGF }=, 749 \\
\text { CFI }=, 897 \\
\text { RMSEA }=, 160 \\
(, 123-, 197) \\
\alpha \text { de Cronbach }=, 859 \\
\rho_{\mathrm{c}}=, 864 \\
\text { AVE }=, 495,\end{array}$} & ,389 & \multirow{7}{*}{$\begin{array}{c}X^{2}=68,202 \\
\text { P-valor }=, 000 \\
\text { GFI }=, 885 \\
\text { AGF }=, 769 \\
\text { CFI }=, 898 \\
\text { RMSEA }=, 158 \\
(, I 2 I-, 195) \\
\alpha \text { de Cronbach }=, 860 \\
\rho_{c}=, 865 \\
\text { AVE }=, 495\end{array}$} \\
\hline 2. & $\begin{array}{l}\text { Se comparten ideas, información y otros } \\
\text { recursos con los proveedores/clientes } \\
\text { clave }\end{array}$ & ,399 & & 415 & \\
\hline 3. & $\begin{array}{l}\text { Existen equipos formales con los pro- } \\
\text { veedores/clientes clave para integrar } \\
\text { procesos }\end{array}$ & ,649 & & ,671 & \\
\hline 4. & $\begin{array}{l}\text { Se realiza una planificación conjunta con } \\
\text { los proveedores/clientes clave para anti- } \\
\text { cipar y resolver problemas }\end{array}$ &, 714 & & ,755 & \\
\hline 5. & $\begin{array}{l}\text { Se establecen objetivos conjuntos con } \\
\text { los proveedores/clientes clave }\end{array}$ & ,868 & & ,840 & \\
\hline 6 & $\begin{array}{l}\text { Se desarrollan responsabilidades conjun- } \\
\text { tas con los proveedores/clientes clave }\end{array}$ & ,878 & & ,883 & \\
\hline 7. & $\begin{array}{l}\text { Se toman decisiones conjuntas con los } \\
\text { proveedores/clientes clave para mejorar } \\
\text { la eficiencia de los costes }\end{array}$ &, 825 & & ,797 & \\
\hline
\end{tabular}

la Chi-cuadrado y su p-valor, el RMSEA, el AGF o la varianza extraída tampoco aseguran la validez convergente de los ítems. Esto nos llevaría a plantear una depuración mediante nuevos análisis de naturaleza confirmatoria.

Como podemos observar, son los tres primeros ítems ( 1 . Existen equipos informales con los proveedores/clientes clave para integrar procesos, 2 . Se comparten ideas, información y otros recursos con los proveedores/clientes clave y 3. Existen equipos formales con los proveedores/clientes clave para integrar procesos) los que tienen cargas factoriales inferiores a 0,7 en ambas escalas, por lo que iremos eliminándolos de forma progresiva para conseguir nuestro objetivo.

En un segundo AFC pasamos a eliminar el ítem 2, que tiene menor carga en la escala de proveedores, y el ítem I para la de clientes. Los resultados siguen sin ofrecer un buen ajuste, ya que, si bien algunos indicadores globales mejoran, no todos cumplen los criterios deseados. De esta forma, hemos procedido sucesivamente con el resto de ítems, hasta comprobar que no obtenemos ningún resultado parcial adecuado, por lo que hemos tenido que depurar los ítems I, 2 y 3 en ambas escalas iniciales, tal y como mostramos en la Tabla 5.

Como se observa, las cargas factoriales estandarizadas de los cuatro ítems finales cumplen los criterios requeridos para contribuir a formar el factor, excepto el ítem 4 de la escala de proveedores, si bien su valor se puede considerar cercano al aceptable. Por otro lado, respecto a los parámetros globales de las escalas vemos que el valor de la Chi-cuadrado ha disminuido mucho en ambas escalas (proveedores y clientes) y su p-valor obtiene un valor superior a 0,05, lo que es deseable. Respecto a los indicadores GFI, AGF y CFI, todos ellos tienen valores superiores a 0,9 y el RMSEA cumple que el cero se encuentra dentro del intervalo, así como el valor de la varianza extraída, que en ambas escalas es superior a 0,5 tal y como recomiendan Fornell y Larcker (1981). De esta forma, podemos asegurar que las escalas cumplen con la validez convergente, por lo que sí hemos en- 
Tabla 5

Análisis factorial de las cuatro actividades de integración e indicadores de fiabilidad

\begin{tabular}{|c|c|c|c|c|c|}
\hline & \multirow[b]{2}{*}{ Análisis factorial con 4 ítems } & \multicolumn{2}{|c|}{$\begin{array}{l}\text { Integración externa } \\
\text { con proveedores }\end{array}$} & \multicolumn{2}{|c|}{ Integración externa con clientes } \\
\hline & & $\begin{array}{c}\text { Carga } \\
\text { factorial }(\lambda)\end{array}$ & Fiabilidad & $\begin{array}{c}\text { Carga } \\
\text { factorial }(\lambda)\end{array}$ & Fiabilidad \\
\hline 4. & $\begin{array}{l}\text { Se realiza una planificación conjunta con } \\
\text { los proveedores/clientes clave para anti- } \\
\text { cipar y resolver problemas }\end{array}$ & ,676 & \multirow{4}{*}{$\begin{array}{c}X^{2}=4,773 \\
\text { P-valor }=, 092 \\
\text { GFI }=, 985 \\
\text { AGF }=, 925 \\
\text { CFI }=, 993 \\
\text { RMSEA }=, 095 \\
(, 000-, 207) \\
\alpha \text { de Cronbach }=, 889 \\
\rho_{c}=, 893 \\
\text { AVE }=, 677\end{array}$} & ,730 & \multirow{4}{*}{$\begin{array}{c}X^{2}=1,000 \\
\text { P-valor }=, 607 \\
\text { GFI }=, 997 \\
\text { AGF }=, 984 \\
\text { CFI }=1,000 \\
\text { RMSEA }={ }^{\prime} 000 \\
(, 000-, \mid 129) \\
\alpha \text { de Cronbach }=, 889 \\
\rho_{c}=, 89 \mid \\
\text { AVE }=, 673\end{array}$} \\
\hline & $\begin{array}{l}\text { Se establecen objetivos conjuntos con } \\
\text { los proveedores/clientes clave }\end{array}$ & 897 & & ,862 & \\
\hline 6. & $\begin{array}{l}\text { Se desarrollan responsabilidades conjun- } \\
\text { tas con los proveedores/clientes clave }\end{array}$ & ,881 & & ,884 & \\
\hline 7. & $\begin{array}{l}\text { Se toman decisiones conjuntas con los } \\
\text { proveedores/clientes clave para mejorar } \\
\text { la eficiencia de los costes }\end{array}$ & 820 & &, 798 & \\
\hline
\end{tabular}

contrado una correspondencia entre la construcción conceptual extraída de la revisión teórica y la escala propuesta para cuantificarla.

Para finalizar el apartado de resultados, señalar que los indicadores obtenidos en las escalas para el alpha de Cronbach, así como para la fiabilidad compuesta de las mismas nos aseguran que las escalas son fiables (Bagozzi yYi, 1988). De esta forma, hemos conseguido validar las escalas propuestas para la integración externa con proveedores y clientes con un total de 4 ítems.

\section{Conclusiones}

La literatura sobre cadena de suministro enfatiza el concepto de la integración empresarial como un elemento clave para la obtención de ventajas competitivas por parte de las empresas, e implica la interrelación tanto con proveedores como con clientes. Sin embargo, no encontramos un acuerdo general a la hora de medir este concepto, por lo que mediante este trabajo hemos pretendido contribuir al debate mediante la propuesta y validación de dos escalas de medida para la integración externa, una para proveedores y otra para clientes.

Para cumplir con el objetivo propuesto hemos llevado a cabo en primer lugar una revisión bibliográfica de aquellos trabajos considerados como principales aportaciones sobre el concepto de la integración externa.

A continuación hemos propuesto una escala de medida para proveedores y clientes integrada por 7 ítems, que nos ha permitido una mejor comprensión del concepto y que ha sido necesaria depurar mediante sucesivos análisis factoriales confirmatorios. El resultado de este análisis ha proporcionado apoyo empírico a otras investigaciones realizadas sobre la integración externa, como por ejemplo Giménez y Ventura (2005), Narasimhan y Das (200I), Rosenzweig et al. (2003), Rodrigues et al. (2004), Stank et al. (200 I) o Li et al. (2006). Sin embargo, la escala final, tanto para proveedores como para clientes, ha sido validada únicamente con 4 de los 7 ítems, en concreto planificación conjunta, objetivos conjuntos, responsabilidades conjuntas y decisiones conjuntas.

Los 3 ítems que el análisis ha depurado son los de existencia de equipos informales, información e ideas compartidas y equipos formales. Si hacemos una reflexión sobre éstos podemos pensar que este hecho puede ser debido bien a la propia redacción de los mismos, bien a la percepción de los encuestados hacia estos conceptos. Sin embargo, podemos considerar que otros conceptos de la escala como la existencia de objetivos compartidos y la planificación conjunta implicarían de forma explícita la existencia de equipos formales, así como las ideas e información compartida. Por otro lado, la existencia de equipos informales es más complicada de percibir en los procesos de integración y precisaría en todo caso de un análisis más profundo.

En definitiva, consideramos que el objetivo propuesto en este trabajo de investigación ha sido cubierto mediante la propuesta y depuración final de las escalas. Por lo que resulta un primer paso para 
continuar con nuestra investigación futura donde propondremos modelos teóricos que nos permitan estudiar la influencia de la integración externa sobre otras variables como el resultado empresarial. Si bien hay trabajos previos (Frohlich y Westbrook, 2001 ; Chen y Paulraj, 2004) pretendemos incorporar nuevas variables, como por ejemplo la confianza en la relación.

El trabajo cuenta a su vez con algunas limitaciones que cabe señalar. Así por ejemplo, la integración externa se ha estudiado en un momento del tiempo, pero hay que tener en cuenta que es el resultado del transcurso de las relaciones desarrolladas hasta ese punto. Cabe a su vez plantear la validación de la escala en otros sectores industriales, quizá no tan concentrados geográficamente como el sector textil valenciano. Esto nos permitiría observar si las escalas son robustas en otros contextos. En este caso, deberíamos desarrollar un proceso de invarianza métrica para comprobar si los resultados son consistentes en ambos grupos y si los conceptos que se miden se interpretan de la misma manera (Steemkamp y Baumgartner, 1998).

\section{Agradecimientos}

Esta investigación ha sido financiada por el Ministerio de Educación y Ciencia, Plan Nacional de I+D+i (2007-20 I0), proyecto «El Distrito Industrial: el impacto del Capital Social sobre la Gestión de la Cadena de Suministro» (SEJ2007-62876/ECON).

\section{Bibliografía}

ANDERSON, J.C. y GERBING, D.W. (1988). «Structural equation modelling in practice: A review and recommended two-step approach». Psychological Bulletin, 103 , pp. 4 | I-423.

BAGCHI, P.K. y SKJOETT-LARSEN,T. (2005). «Supply chain integration: a European survey». The International Journal of Logistics Management, 16 (2), pp. 275-294.

BAGOZZI, R.P.yYI,Y. ( 1988). «On the evaluation of structural equations models». Journal of the Academy of Marketing Science, 16 ( I), pp. 74-94.

BOLLEN, K.A. (1989). Structural equations with latent variables, New York: John Wiley \& Sons.

CHEN, I.J. y PAULRAJ, A. (2004). «Towards a theory of Supply Chain Management: The Constructs and measurements». Journal of Operations Management, 22, pp. | |9-।50.
CHRISTOPHER, M. (1998). Logistics and Supply Chain Management: Strategies for reducing cost and improving service, Financial Times Pitman Publishing.

CUELLO DE ORO CELESTINO, D.J., DELGADO ESTIRADO, L.M. y FOSSAS OLALLA, M. (2003). «Una aplicación del modelo Intelect: estudio del capital relacional entre Espack y Ford España». Dirección y Organización, 29, pp. 29-I33.

DEAN, J.W. y SNELL, S.A. (199I). «Integrated manufacturing and job design: moderating effects of organizational inertia». Academy of Management Journal, 34 (4), pp. 776-804.

DROGE, C., JAYARAM, J. y VICKERY, S.K. (2004). «The effects of InternalVersus External Integration practices on time-based performance and overall firm performance». Journal of Operations Management, 22, pp. 557-573.

ELLINGER, A., DAUGHERTY, P. y KELLER, S. (2000). «The relationship between marketing/ logistics interdepartmental integration and performance in U.S. manufacturing firms: An empirical study». Journal of Business Logistics, 21 ( I), pp. I -22.

FABBE-COSTES, N. y JAHRE, M. (2007). «Supply Chain Integration Improves Performance:The Emperor's New Suit?». International Journal of Physical Distribution \& Logistics Management, 37( I0), pp. 835-855.

FORNELL, C. y LARCKER, D.F. (|98I). «Evaluating structural equations models with unobservable variables and measurement error»». Journal of Marketing Research, | 8 , pp. 39-50.

FROHLICH, M.T. y WESTBROOK, R. (200I). «Arcs of integration: an international study of supply chain strategies». Journal of Operations Management, 19, pp. I 85-200.

GIMENEZ, C. y VENTURA, E. (2003). «Supply Chain Management as a competitive advantage in the Spanish grocery sector»». The International Journal of Logistics Management, I 4 ( I), pp. 77-88.

GIMENEZ, C. y VENTURA, E. (2005). «Logistics-Productions, Logistics-Marketing and External Integration, their impact on performance». International Journal of Operation \& Production Management, 25 ( I ), pp. 20-38.

HAIR, J.F., ANDERSON, R.E., TATHAM, R.L. y BLACK,W.C. ( 1999). Análisis Multivariante. Madrid: Ed. Prentice Hall.

JORESKOG, K.G. y SORBOM, D. (1993). LISREL 8:Structural Equation Modeling with the SIMPLIS Command Language, Chicago: Ed. Scientific Software International.

KINNEAR, J.C.y TAYLOR, J.R. ( 1995). Investigación de mercados: un enfoque aplicado, Madrid: Ed. McGraw Hill.

LEJEUNE, M.A. y YAKONA, N. (2005). «On characterizing the 4C's in supply chain management». Journal of Operations Management, 23, pp. 8I-100. 
LI, S., RAGU-NATHAN, B., RAGU-NATHAN, T.S. y RAO, S.S. (2006). «The impact of supply chain management practices on competitive advantage and organizational performance». Omega, 34, pp. 107- 124.

MENTZER, J.T., DEWITT, W., KEEBLER, J.S., MIN, S., NIX, N.W., SMITH, C.D. y ZACHARIA, Z.G. (200I). «Defining supply chain management». Journal of Business Logistics, 22 (2), pp. I-25.

MILLER, J.G. y ROTH, A.V. ( 1994). «A taxonomy of manufacturing strategies». Management Science, 40 (3), pp. 285-304.

MITTERMAYER, H. y RODRÍGUEZ MONRO, C. (2009). «Evaluación basada en la simulación de enfoques de gestión de la cadena de suministro (des)centralizada». Dirección y Organización, 39, pp. 62-70.

NARASIMHAN, R. y DAS, A. (200I). «The impact of purchasing integration and practices on manufacturing performance». Journal of Operations Management, 19. pp. 593-609.

RODRIGUES, A.M., STANK, T.P.y LYNCH, D.F. (2004). «Linking Strategy, Structure, Process, and Performance in Integrated Logistics». Journal of Business Logistics, 25(2), pp. 65-94.

ROSENZWEIG, E.D., ROTH, A.V. y DEAN Jr., J.W. (2003). «The influence of an integration strategy on competitive capabilities and business performance: An exploratory study of consumer products manufacturers». Journal of Operations Management, 21, pp. 437-456.

SANCHEZ PEREZ, M. (1997). «Las relaciones interorganizacionales en el canal de comercialización». Colección Monografías de Ciencias Económicas y Jurídicas, n. ${ }^{\circ} 10$, Servicio de Publicaciones de la Universidad de Almería, Almería.

SHIN, H., COLLIER, D.A. y WILSON, D.D. (2000). «Supply management orientation and supplier / buyer perfor- mance». Journal of Operations Management, I 8 (3), pp. 317-333.

STANK,T.P., KELLER, S.B. y CLOSS, D.J. (200 I-2002). «Performance Benefits of Supply Chain Logistical Integration». Transportation Journal, 4 I (2/3), pp. 32-46.

STANK, T.P., GOLDSBY,T.J., VICKERY, S.K. y SAVITSKIE, K. (2004). «Logistics Service performance: Estimating its influence on market share». Journal of Business Logistics, 24 ( I), pp. 27-55.

STEENKAMP, J.B.E.M. y BAUMGARTNER, H. ( 1 998). «Assessing Measurement Invariance in Cross-National Consumer Research». Journal of Consumer Research, 25 (I), pp. 78-90.

STEVENS, G.C. (1 989). «Integrating the Supply Chain». International Journal of Physical Distribution and Materials Management, 19 (8), pp. 3-8.

STOCK, G.N., GREIS, N.P. y KASARDA, J.D. ( 1998). «Logistics, strategy and structure: A conceptual framework». International Journal of Operations and Production Management, 18 ( I), pp. 37-52.

STUART F.I. (1997). «Supply-chain strategy: organizational influence through supplier alliances». British Academy of Management, 8 (3), pp. 223-236.

VALLET-BELLMUNT,T. (20 I 0). «Las relaciones en la cadena de suministro no son tan peligrosas». Universia Business Review, 26 (2), pp. 12-33.

VAN DER VAART,T.y VAN DONK, D.P. (2008). «A critical review of survey-based research in supply chain integration». International Journal of production Economics, I । I, pp. 42-55.

VICKERY, S.K., DROGE, C., STANK, T.P., GOLDSBY,T.J. y MARKLAND, R.E. (2004). «The Performance Implications of Media Richness in a Business-to-Business Service Environment: Direct Versus Indirect Effects». Management Science, 50 (8), pp. I I06- I I 19. 